\title{
Acknowledgment of Reviewers
}

In addition to members of the Editorial Board, the individuals listed below provided constructive critical reviews of one or more manuscripts during the past year. Those who reviewed their first manuscript near the end of the year will be recognized in next year's acknowledgments. Appreciation for their valuable help to authors and the journal is expressed to the following reviewers:

\begin{tabular}{|c|c|c|}
\hline G. Abad & M. Cambra & L. J. du Toit \\
\hline H. Abbas & E. Cantonwine & P. Duan \\
\hline J. Adaskaveg & O. Carisse & F. M. Dugan \\
\hline P. Agudelo & A. Carlucci & L. Duncan \\
\hline M. C. Aime & L. Carris & N. Duran-Vila \\
\hline M. Akos & R. Cartwright & D. M. Eastburn \\
\hline C. Allen & F. Caruso & K. Eastwell \\
\hline T. Alle & N. Castilla & J. Edwards \\
\hline R. Almeida & C. J. Chang & N. Edwards \\
\hline L. A. Alvarez & Y.-C. Chang & D. S. Egel \\
\hline A. M. Alvarez & N. Charlton & J. D. Eisenback \\
\hline S. Ambros & A. Chase & A. Elling \\
\hline J. Anderson & T. Chase & M. Elliott \\
\hline D. Andrivon & G. Chastagner & M. Ellis \\
\hline J. A. Asselin & P. Chaverri & W. H. Elmer \\
\hline F. M. Assis Filho & F. Chen & J. Elphinstone \\
\hline M. Babadoost & W. Chen & S. Enebak \\
\hline P. Backman & P. Chiembsombat & L. Epstein \\
\hline G. Bai & B. Christ & D. Errampalli \\
\hline J. Bakonyi & K.-R. Chung & T. Evans \\
\hline S. Banniza & A. Ciancio & K. Everts \\
\hline J. Barak & M. Cilia & B. Falk \\
\hline H. S. Bariana & E. Civerolo & M. Farman \\
\hline O. Becker & C. A. Clark & J. Farrar \\
\hline J. L. Beckerman & M. Clayton & R. Ferreira \\
\hline A. Belisario & W. Cline & S. Ferreira \\
\hline R. Bennett & M. D. Coffey & D. M. Ferrin \\
\hline M. Benson & C. Colburn & T. Fetch, Jr. \\
\hline M. Berbegal & P. Colyer & G. Filip \\
\hline A. Bergamin Filho & J. C. Comstock & J. Fletcher \\
\hline G. Bergstrom & D. Cooksey & R. Flores \\
\hline D. Berner & D. R. Cooley & T. Forge \\
\hline A. M. Berrie & W. Copes & B. Fortnum \\
\hline H. Bertaccini & J. Correll & S. Fraedrich \\
\hline P. Bertrand & C. Cowger & G. Franc \\
\hline A. R. Biggs & K. Cox & J. Freitas-Astúa \\
\hline E. Biosca & R. Creamer & R. French \\
\hline L. L. Black & J. A. Crouch & M. Fuchs \\
\hline C. Blanco & W. T. Crow & D. L. Funnell \\
\hline C. C. Block & M. A. Cubeta & L. M. Gullino \\
\hline B. Bluhm & A. Culbreath & E. Gamliel-Atinsky \\
\hline C. Bock & D. Cuppels & C. Garzon \\
\hline P. Bonello & J. V. da Graça & D. Gaudet \\
\hline J. Borneman & J. Damicone & E. E. Gbur \\
\hline S. Bost & V. D. Damsteegt & A. Gera \\
\hline R. Bostock & M. Daub & T. German \\
\hline R. Bowden & M. L. Daughtrey & A. J. Gevens \\
\hline K. L. Bowen & J.-H. J. Daugrois & R. Gilbertson \\
\hline J. Bowers & D. Davis & D. Glawe \\
\hline C. Bragard & S. H. De Boer & M. L. Gleason \\
\hline M. P. Braverman & H. de Gruyter & B. Goates \\
\hline T. Brenneman & C. De Vallavielle-Pope & A. J. González \\
\hline R. Briddon & M. Deadman & S. Goodwin \\
\hline W. L. Bruckart & L. E. del Rio Mendoza & C. Gouk \\
\hline B. Bruton & T. Denny & J. Graham \\
\hline C. T. Bull & M. Dewdney & D. Gray \\
\hline R. Bulluck & M. Dickinson & C. Greer \\
\hline R. Buonaurio & R. Dietzgen & H. M. Griffiths \\
\hline T. I. Burgess & R. Dill-Macky & M. Grisham \\
\hline G. Burow & L. Domier & M. Groenwald \\
\hline L. Burpee & R. Donahoo & D. Groth \\
\hline T. Burr & E. Dor & N. J. Grünwald \\
\hline
\end{tabular}


W. D. Gubler

N. Gudmestad

T. Gulya

J. M. Halbrendt

B. Hall

D. Hall

S. Hambleton

P. B. Hamm

R. W. Hammond

E. Hansen

L. Hanson

J. Hao

R. Harding

P. F. Harmon

T. Harrington

N. A. Harrison

J. Hartung

B. Henricot

D. Hershman

J. D. Hill

C. A. Hoepting

S. Hogenhout

G. E. Holcomb

C. Hollingsworth

C. Hong

D. Hopkins

K. Horst

M. Hovmoller

J. Hoy

$\mathrm{S}$. Hu

Q. Huang

D. M. Huber

D. Huberli

T. Hughes

R. M. Hunger

J. Hwang

N. S. Iacobellis

D. M. Ingram

A. K. Inoue-Nagata

M. S. Irey

T. Isakeit

S. A. Isard

H. Ishii

T. Jackson

E. W. Jackson

W. R. Jacobi

M. Jacquemond

D. James

F.-J. Jan

W. J. Janisiewicz

D. J. Jardine

J. Jayasankar

S. N. Jeffers

P. Ji

Y. Jia

M. I. Jimenez

M. Jimenez Gasco

Y. Jin

K. Johnson

D. A. Johnson

C. S. Johnson

J. Jones

R. A. C. Jones

C. Jorda

R. Jordan

J. Juzwik

I. Kamenova

J. E. Kaminski

L. Kanetis

G. S. Karaoglanidis
H.-H. Kassemeyer

L. Keith

R. Kemerait

J. P. Kerns

J. Kerrigan

M. F. Khan

L. Kiss

M. Klich

S. J. Klosterman

K. Klotz

L. Kohn

S. T. Koike

J. Kolmer

J. Kotcon

C. Kousik

J. Kreuze

J. E. Kurle

J. LaMondia

R. Labuda

D. H. Lambert

B. Landa del Castillo

P. Landschoot

M. Langham

D. Langston

R. Larkin

R. Larsen

B. A. Latorre

L. Leandro

F. Lee

I.-M. Lee

S. L. Lenardon

K. Leonard

J. Leslie

D. J. Lewandowski

R. Lewellen

K. Lewis

W. Li

Y. Li

A. Lichter

L. W. Liefting

K.-S. Ling

C. Little

H.-Y. Liu

X. Z. Liu

B. Lockhart

L. Lombard

M. M. Lopez

S. A. Lopes

R. Loria

S. Lu

Y. Luan

O. Lukow

J. E. Lundquist

P. Lunello

Y. Luo

E. Morlejo

W. L. MacDonald

L. V. Madden

G. Magnano di San Lio

W. F. Mahaffee

N. Mahfoudhi

C. Malmstrom

D. Malvick

J. Marois

C. J. Maroon-Lango

D. Marshall

G. P. Martelli

S. B. Martin

R. Martyn

S. Masoud
M. E. Matheron

D. Mathews

P. Mauk

G. Mbofung

B. D. McCallum

G. McCollum

J. D. McCreight

M. T. McGrath

E. H. C. McKenzie

M. P. McMullen

B. McSpadden Gardener

E. Medrano

H. Melakeberhan

A. Mengistu

J. Menzies

S. Meyer

T. J. Michailides

A. Mila

S. A. Miller

C. Mitchell

T. Mitchell

M. T. Mmbaga

G. W. Moorman

E. Moriones

C. Morris

M. M. Mota

B. Moury

J. D. Mueller

D. S. Mueller

J. Mullen

R. P. Mulrooney

G. Munkvold

J. F. Murphy

T. Murray

M. Newcomb

M. Nakhla

J. Navas-Castillo

B. Nelson

R. Nelson

S. Nelson

G. Newcombe

H. Ngugi

X. Nie

R. Niks

C. Nischwitz

K. A. Nishijima

M. Nita

N. Nitzan

J. Noe

D. Norman

M. Nuti

K. O'Donnell

$\mathrm{K}$. Ong

N. Osterbauer

M. Ostry

R. A. Owens

O. Pruvost

B. Padgett

A. Palacio-Bielsa

M. E. Palm

A. J. Palmateer

Y.-B. Pan

H. R. Pappu

J. Parke

J. K. Pataky

T. C. Paulitz

G. Payne

W. Pedersen

Z. Perez-Egusquiza

K. Pernezny 


\begin{tabular}{|c|c|c|}
\hline K. Perry & R. Schneider & A. Trapero-Casas \\
\hline D. M. Persley & C. Schoch & L. P. Tredway \\
\hline P. Peterson & B. Schroeder & E. Turco \\
\hline G. L. Peterson & T. Schubert & I. E. Tzanetakis \\
\hline E. Petit & H. F. Schwartz & J. Y. Uchida \\
\hline A. Phillips & S. Scott & W. Uddin \\
\hline G. Pietersen & G. Secor & J. R. Urbez-Torres \\
\hline A. T. Ploeg & K. W. Seebold, Jr. & J. Valkonen \\
\hline M. L. Polek & D. L. Seifers & R. Valverde \\
\hline G. Polizzi & M. Serdani & M. Verbeek \\
\hline J. Polston & D. Sether & J. Th. J. Verhoeven \\
\hline C. A. Powell & S. F. Shamoun & J. A. Verreet \\
\hline Z. A. Pretorius & D. Shaw & A. M. Vettraino \\
\hline J. Prince & J. Sherwood & G. Vidalakis \\
\hline D. Prusky & D. Shew & P. Vincelli \\
\hline J. W. Pscheidt & A. Shi & H. Voglmayr \\
\hline Z. Punja & W. B. Shim & R. R. Walcott \\
\hline L. Pusey & P. L. Sholberg & G. Walker \\
\hline M. L. Putnam & D. Shtienberg & M. Walter \\
\hline W. Qiu & P. Sieburth & G.-L. Wang \\
\hline L. M. Quesada-Ocampo & D. Six & N. Wang \\
\hline M. Rahman & A. Skelton & X. Wang \\
\hline R. Raid & B. Slippers & L. A. Wanner \\
\hline S. N. Rampersad & R. Smiley & C. Y. Warfield \\
\hline K. Rane & M. Smith & S. N. Wegulo \\
\hline G. Rauscher & D. L. Smith & J. Weiland \\
\hline N. A. Rayapati & E. Souza & D. M. Weller \\
\hline S. Reitz & R. Spotts & C. Wellings \\
\hline S. Rideout & A. Sreedharan & R. Welliver \\
\hline C. Ridout & S. Sreenivasaprasad & R. Wick \\
\hline E. Riga & R. R. Stange & W. F. Wilcox \\
\hline J. B. Ristaino & M. Stanghellini & D. K. Willis \\
\hline D. Rizzo & G. Stanosz & J. M. Windes \\
\hline J. Robb & D. C. Stenger & S.-M. Wong \\
\hline P. Roberts & K. Stevenson & A. Wood \\
\hline A. Robertson & V. Stockwell & S. Woodward \\
\hline N. L. Robertson & J. Stone & J. Worrall \\
\hline D. M. Rochon & S. E. Strelkov & A. Wrather \\
\hline A. Roelfs & R. Sturrock & B. M. Wu \\
\hline M. Romberg & K. Subbarao & A. Wyenandt \\
\hline J. Romero & S. Subbotin & J.-R. Xu \\
\hline D. A. Rosenberger & D. Sugar & L. Yakabe \\
\hline E. Rosskopf & T. Sugimoto & K. Yoder \\
\hline C. Rothrock & B. Summerell & D. S. Yohalem \\
\hline J. Roux & G. Sundin & R. K. Yokomi \\
\hline R. Rowe & T. Sutton & N. Yoshikawa \\
\hline D. Rubales & L. J. Szabo & G. Yuen \\
\hline J. C. Rupe & S. Tatineni & N. Zhang \\
\hline K. H. Ryu & J. C. Tello Marquina & Z. Zhang \\
\hline S. Sabanadzovic & J. A. Thies & S. Zhong \\
\hline L. Salazar & T. Thomidis & E. Zhou \\
\hline P. Saldarelli & L. W. Timmer & X.-G. Zhou \\
\hline G. Samuels & P. Timper & T. A. Zitter \\
\hline S. Sanogo & T. Toda & M. van Iersel \\
\hline T. Sasaya & S. A. Tolin & C. Vera Cruz \\
\hline N. Schaad & M. Tomaso-Peterson & S. von Bargen \\
\hline C. Schardl & P. W. Tooley & S. von Bodman \\
\hline H. Scherm & I. Toth & \\
\hline A. C. Schilder & F. Trail & \\
\hline
\end{tabular}

